\title{
Biomarker Identification and Pathway Analysis by Serum Metabolomics of Lung Cancer
}

\author{
Yingrong Chen, ${ }^{1}$ Zhihong Ma, ${ }^{1}$ Lishan Min, ${ }^{1}$ Hongwei Li, ${ }^{2}$ \\ Bin Wang, ${ }^{3}$ Jing Zhong, ${ }^{1}$ and Licheng Dai ${ }^{1,4}$ \\ ${ }^{1}$ Huzhou Key Laboratory of Molecular Medicine, Huzhou Central Hospital, Huzhou, Zhejiang 313000, China \\ ${ }^{2}$ Cardiothoracic Surgery, Huzhou Central Hospital, Huzhou, Zhejiang 313000, China \\ ${ }^{3}$ Respiratory Medicine, Huzhou Central Hospital, Huzhou, Zhejiang 313000, China \\ ${ }^{4}$ School of Laboratory Medicine and Life Science, Wenzhou Medical University, Wenzhou, Zhejiang 325035, China
}

Correspondence should be addressed to Licheng Dai; dlc@hzhospital.com

Received 11 February 2015; Revised 23 March 2015; Accepted 27 March 2015

Academic Editor: Xin-yuan Guan

Copyright (c) 2015 Yingrong Chen et al. This is an open access article distributed under the Creative Commons Attribution License, which permits unrestricted use, distribution, and reproduction in any medium, provided the original work is properly cited.

\begin{abstract}
Lung cancer is one of the most common causes of cancer death, for which no validated tumor biomarker is sufficiently accurate to be useful for diagnosis. Additionally, the metabolic alterations associated with the disease are unclear. In this study, we investigated the construction, interaction, and pathways of potential lung cancer biomarkers using metabolomics pathway analysis based on the Kyoto Encyclopedia of Genes and Genomes database and the Human Metabolome Database to identify the top altered pathways for analysis and visualization. We constructed a diagnostic model using potential serum biomarkers from patients with lung cancer. We assessed their specificity and sensitivity according to the area under the curve of the receiver operator characteristic (ROC) curves, which could be used to distinguish patients with lung cancer from normal subjects. The pathway analysis indicated that sphingolipid metabolism was the top altered pathway in lung cancer. ROC curve analysis indicated that glycerophospho-Narachidonoyl ethanolamine (GpAEA) and sphingosine were potential sensitive and specific biomarkers for lung cancer diagnosis and prognosis. Compared with the traditional lung cancer diagnostic biomarkers carcinoembryonic antigen and cytokeratin 19 fragment, GpAEA and sphingosine were as good or more appropriate for detecting lung cancer. We report our identification of potential metabolic diagnostic and prognostic biomarkers of lung cancer and clarify the metabolic alterations in lung cancer.
\end{abstract}

\section{Introduction}

Lung cancer is one of the most common cancers worldwide; the prognosis for many patients with lung cancer remains poor. The high mortality and poor prognosis of lung cancer are mainly due to the difficulty of early diagnosis. If patients were diagnosed early, the average 5-year survival rate could be as high as $85 \%$ [1]. The development of molecular biology has enabled tumor markers to become a common means of diagnosing cancer. The most widely used lung cancer biomarkers are carcinoembryonic antigen (CEA), cancer antigen 125, cytokeratin 19 fragment (CYFRA21-1), and neuron-specific enolase [2]. However, no validated tumor marker is sufficiently accurate to be useful for diagnosis to date. Therefore, searching for novel diagnostic biomarkers of lung cancer remains difficult.

Metabolomics is a powerful quantitative measurement of low-molecular weight metabolites of an organism at a specified time in specific environmental conditions. Fundamental analytical techniques are used to probe the chemical fingerprint of samples and are an effective tool for screening biomarkers $[3,4]$, diagnosis $[5,6]$, and biological pathway characterization [7], specifically and accurately correlating a particular pathway and hence any biomarkers in that pathway with the disruption. Using a more precise selection process for candidate marker identification, metabolomics increases the likelihood of validation of candidate biomarkers in subsequent prospective validation studies [8-10]. 
The approach also enhances the ability of researchers to use the metabolomic data collected from the biomarker discovery phase to gain insight into disease biology.

Metabolomic studies in lung cancer samples have generally employed techniques such as nuclear magnetic resonance [11], high-performance liquid chromatography/mass spectrometry (HPLC/MS and LC/MS/MS) [12], and gas chromatography/MS (GC/MS). HPLC coupled with quadrupole time-of-flight MS (HPLC-Q-TOF/MS) is widely used in metabolomics because it yields accurate qualitative analysis. Given its high sensitivity, peak resolution, and reproducibility, GC/MS is robust metabolomic tool also widely used in metabolite identification and quantification [13].

Previously [14], we used LC-Q-TOF/MS and GC/MS to compare the metabolite profiles of serum from preoperative patients with lung cancer (PRLC), postoperative patients with lung cancer (POLC), and healthy volunteers (controls). We characterized differences in the metabolomic profiles of the three groups using multivariate statistical analyses: principal components analysis (PCA) and partial least squares discriminant analysis (PLS-DA). From the pattern recognition results, we identified ten potential metabolic biomarkers for lung cancer diagnosis.

In this study, we analyzed the construction, interaction, and pathways of potential lung cancer biomarkers using metabolomics pathway analysis (MetPA) based on the Kyoto Encyclopedia of Genes and Genomes (KEGG) database and Human Metabolome Database to identify the top altered pathways for analysis and visualization. We constructed a diagnostic model using potential serum biomarkers from patients with lung cancer. We assessed their classification performance (specificity and sensitivity) using the area under the curve (AUC) of the receiver operator characteristic (ROC) curve, which might be used to distinguish patients with lung cancer from normal subjects.

\section{Materials and Methods}

2.1. Subjects. The Huzhou Central Hospital Ethics Committee approved this prospective study; we obtained informed consent from each participant. Serum samples were collected from 30 healthy volunteers without serious medical illness (controls) and from 30 patients with lung cancer without previous history of other cancers at Huzhou Central Hospital from January 2012 to January 2013. Patients and volunteers were matched according to sex and age. Of the 30 patients, 15 had adenocarcinoma, 12 had squamous cell carcinoma, and three had large cell carcinoma. The patients were also staged according to the 1997 World Health Organization tumornodes-metastasis (TNM) staging system by Huzhou Central Hospital pathologists: 15 had stage I disease, seven had stage II disease, and eight had stage III disease. All patients had been newly diagnosed and did not receive any form of medical treatment during the sampling period. Preoperative serum was collected before radical correction. Postoperative serum was collected seven days after surgery. Serum was collected from the controls and patients in the morning after fasting. No anticancer agents were administered to the enrolled patients prior to serum collection. Serum samples were collected at the Huzhou Central Hospital Department of Laboratory Medicine; CEA and CYFRA21-1 levels were determined using a Roche COBAS 6000 automated electrochemiluminescence immunoassay analyzer (Roche Diagnostics GmbH; Mannheim, Germany).

2.2. LC-Q-TOF/MS. Serum metabolite profiling was performed on an Agilent 1290 Infinity Liquid Chromatography System (Agilent Technologies, Santa Clara, CA, USA) equipped with a $2.1 \times 100 \mathrm{~mm}$ C18 reverse-phase column with $1.8 \mu \mathrm{m}$ particle size (Waters Corp., Milford, MA, USA). The column was maintained at $40^{\circ} \mathrm{C}$; the injected sample volume was $4 \mu \mathrm{L}$. Gradient conditions were $0-2 \mathrm{~min} 5 \% \mathrm{~B}, 2-17 \mathrm{~min}$ linear gradient from 5 to $95 \%$ B, and $17-19$ min $95 \%$ B. Solvent A was $0.1 \%$ formic acid-water; solvent B was $0.1 \%$ formic acetonitrile. The flow rate was $400 \mu \mathrm{L} / \mathrm{min}$. MS experiments were performed on an Agilent 6530 Accurate-Mass QTOF/MS (Agilent Technologies) equipped with electrospray ionization source. Data for each ionization technique were acquired in positive ion mode. The measurement conditions were capillary voltage $4.0 \mathrm{kV}$, cone voltage $35 \mathrm{kV}$, ion source temperature $100^{\circ} \mathrm{C}$, and vaporizer temperature $350^{\circ} \mathrm{C}$. Nitrogen was used as the nebulizer gas and delivered at a flow rate of $50 \mathrm{~L} / \mathrm{h}$; the desolvation gas (nitrogen) was delivered at a flow rate of $600 \mathrm{~L} / \mathrm{h}$. The scan range was $m / z 50-1000$.

2.3. GC/MS. A $1 \mu \mathrm{L}$ aliquot of derivatized sample was injected splitless into an Agilent 7890A GC system equipped with a $30.0 \mathrm{~m} \times 0.25 \mathrm{~mm}$ i.d. fused-silica capillary column with $0.25-\mu \mathrm{m}$ HP-5 ms stationary phase (Agilent Technologies). The injector temperature was set at $280^{\circ} \mathrm{C}$. Helium was used as the carrier gas at a constant flow rate of $1 \mathrm{~mL} / \mathrm{min}$ through the column. The initial column temperature was $80^{\circ} \mathrm{C}$; after $2 \mathrm{~min}$, the temperature was increased to $320^{\circ} \mathrm{C}$ at a rate of $10^{\circ} \mathrm{C} / \mathrm{min}$ and was held at $320^{\circ} \mathrm{C}$ for $6 \mathrm{~min}$. The column effluent was introduced into the ion source of an Agilent 5975C Mass Selective Detector. The MS quadrupole temperature was $150^{\circ} \mathrm{C}$; the ion source temperature was $230^{\circ} \mathrm{C}$. Masses were acquired at $m / z$ 50-550.

2.4. LC-Q-TOF/MS and GC/MS Data Processing. LC data were acquired and processed using Mass Hunter Qualitative Analysis Software (version B.03.01; Agilent Technologies). The MS analysis system was used to identify metabolites corresponding to those in the METLIN database (http://metlin.scripps.edu).

GC total ion chromatograms and fragmentation patterns were autoacquired using GC/MSD ChemStation software (Agilent Technologies). The mass charge ratios and their abundance were compared with a standard mass chromatogram in the National Institute of Standards and Technology (NIST) mass spectra library using ChemStation, which generated a list of similarities per peak as compared with those in the NIST library.

2.5. Multivariate Data Analysis. Data were exported into SIMCA-P+ 11.0 software (Umetrics AB, Umeå, Sweden) for 
multivariate analysis, that is, PCA and PLS-DA. Data are expressed as the mean \pm SD. An independent $t$-test $(p<0.05)$ was used to determine whether the candidate biomarkers obtained from PLS-DA modeling were statistically significant between groups at univariate analysis level.

2.6. Construction of Metabolic Pathway and Functional Score Analysis. MetPA was used to analyze the construction, interaction, and pathways of the 10 potential lung cancer biomarkers. The MetPA is based on several databases and aids in identifying the top altered pathways for analysis and visualization. In this study, we based the MetPA on the KEGG database (http://www.genome.jp/kegg/) and the Human Metabolome Database (http://www.hmdb.ca/).

2.7. Statistical Analysis. Sample distribution was determined using the Kolmogorov-Smirnov test. Data are expressed as means \pm SD. Analysis of variance was used to analyze the significance of differences between the three groups. We constructed a diagnostic model using the potential serum biomarkers alone or CEA and CYFRA21-1 combined and used the linear discrimination analysis method for analysis. We assessed specificity and sensitivity using the AUC of the ROC curves. All data were analyzed using SPSS version 19.0 (SPSS Inc., Armonk, NY, USA); the significance level was set to $p<0.05$. All $p$ values were two-sided.

\section{Results}

3.1. Identification of Potential Biomarkers. Pattern recognition results identified 10 potential metabolic biomarkers for diagnosing lung cancer (Tables 1 and 2). The serum levels of the potential biomarkers were significantly different in PRLC patients compared with the controls and/or POLC patients. Sphingosine, phosphorylcholine, glycerophospho$\mathrm{N}$-arachidonoyl ethanolamine (GpAEA), $\gamma$-linolenic acid, 9,12-octadecadienoic acid, oleic acid, and serine levels were significantly different in the PRLC patients as compared with those of the controls and POLC patients. Prasterone sulfate, $\alpha$-hydroxyisobutyric acid, and 2,3,4-trihydroxybutyric acid levels were statistically different in PRLC and POLC patients as compared with the controls.

3.2. Comparison of CEA and CYFRA21-1 between Control and Lung Cancer Groups. Table 3 lists the clinical characteristics of the control, PRLC, and POLC groups. Serum CEA and CYFRA21-1 levels of the PRLC group were higher than those of the control and POLC groups.

3.3. Metabolic Pathway and Function Analysis. Pattern recognition analysis of metabolites enabled clear separation of the metabolic profiles of the lung cancer groups and the control group [14]. MetPA was used to perform more detailed analysis of the most relevant lung cancer pathways and networks. MetPA of the potential target metabolic pathways revealed that metabolites detected together were important for the host response to lung cancer. Figure 1 summarizes the pathway analysis, which revealed that the identified metabolites

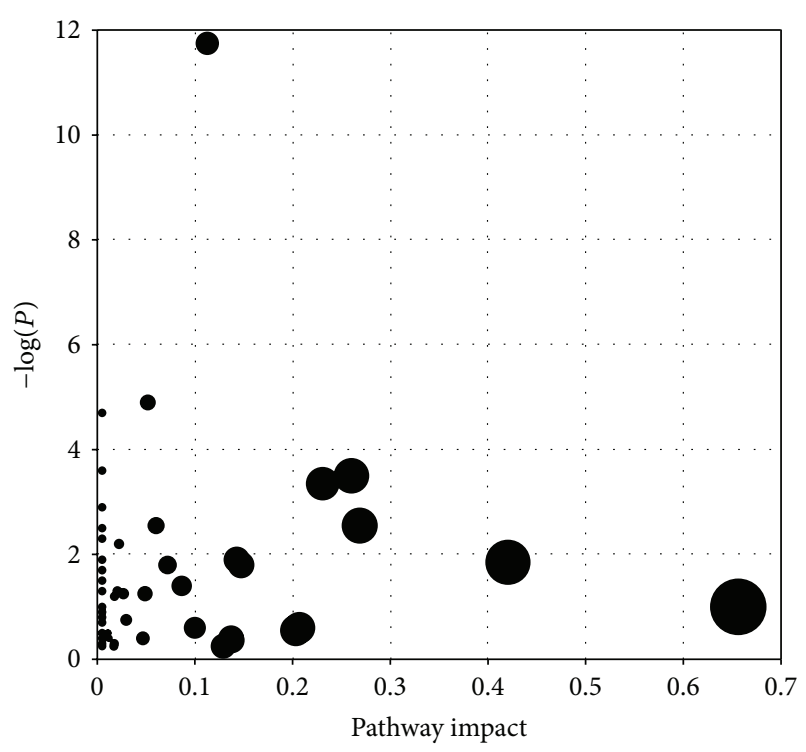

FIGURE 1: Summary of pathway analysis.

important for lung cancer were mainly responsible for the following metabolism pathways: sphingolipid metabolism, glycine, serine, and threonine metabolism, arginine and proline metabolism, galactose metabolism, and linoleic acid metabolism. Table 4 lists the detailed results of the pathway analysis. Figure 2 illustrates the construction of the pathways in detail.

3.4. Diagnostic Value of Potential Serum Biomarkers of Lung Cancer. The ROC curve analysis of potential serum biomarker and other tumor marker (CEA and CYFRA21-1) levels for differentiating the control group from the PRLC groups is shown in Figure 3 (high levels of biomarkers in the PRLC group) and Figure 4 (low levels of biomarkers in the PRLC group). The optimal cutoff points as calculated by Youden's index, sensitivities, specificities, and AUC values are listed in Table 5 (high levels of biomarkers in the PRLC group) and Table 6 (low levels of biomarkers in the PRLC group). The AUC value of GpAEA was 0.983 (95\% confidence interval $[\mathrm{CI}]=0.960-1.000)$. The optimal cutoff point was 1752.6, indicating $96.67 \%$ sensitivity and $90.00 \%$ specificity between the control and PRLC groups. The AUC value of sphingosine was 0.957 (95\% CI $=0.894-1.000)$. The optimal cutoff point was 102.76 , indicating $90.00 \%$ sensitivity and 96.67\% specificity between the control and PRLC groups. The GpAEA and sphingosine AUC were significantly greater than those of the other eight potential biomarkers, CEA, or CYFRA21-1 and had similar diagnostic value to that of CEA, allowing differentiation of the PRLC from the control group. These data indicate that GpAEA and sphingosine are highperformance diagnostic biomarkers of lung cancer, where high GpAEA levels and low sphingosine levels indicate lung cancer risk.

ROC curve analysis of the potential serum biomarker levels for differentiating POLC and PRLC patients is shown in Figure 5 (high levels of biomarkers in the PRLC group) 
TABLE 1: LC-Q-TOF/MS identification of potential serum biomarkers in lung cancer.

\begin{tabular}{lcclcccc}
\hline Number & $\begin{array}{c}\text { Retention } \\
\text { time (min) }\end{array}$ & $m / z$ & Metabolites & Control group & $\begin{array}{c}\text { Relative mass intensity } \\
\text { Preoperative lung } \\
\text { cancer group (PRLC) }\end{array}$ & $\begin{array}{c}\text { Postoperative lung } \\
\text { cancer group (POLC) }\end{array}$ \\
\hline 1 & 9.47 & 368.1655 & Prasterone sulfate & $106.80 \pm 31.70$ & $71.99 \pm 38.72^{*}$ & $50.93 \pm 22.26^{*}$ \\
2 & 11.89 & 299.2816 & Sphingosine & $139.60 \pm 38.75$ & $53.33 \pm 35.95^{* \#}$ & $141.78 \pm 42.42$ \\
3 & 12.17 & 169.0481 & Phosphorylcholine & $72.70 \pm 14.16$ & $133.28 \pm 75.49^{* \#}$ & $82.17 \pm 28.31$ \\
4 & 13.06 & 501.2862 & $\begin{array}{l}\text { Glycerophospho-N-arachidonoyl } \\
\text { ethanolamine }\end{array}$ & $1355.53 \pm 282.89$ & $2722.76 \pm 769.63^{* \#}$ & $1714.79 \pm 399.47$ \\
5 & 16.06 & 278.2241 & $\gamma$-Linolenic acid & $500.34 \pm 204.80$ & $1245.99 \pm 595.41^{* \#}$ & $602.06 \pm 226.28$ \\
\hline
\end{tabular}

${ }^{*}$ Compared with control group, $p<0.05 ;{ }^{*}$ compared with postoperative lung cancer group, $p<0.05$.

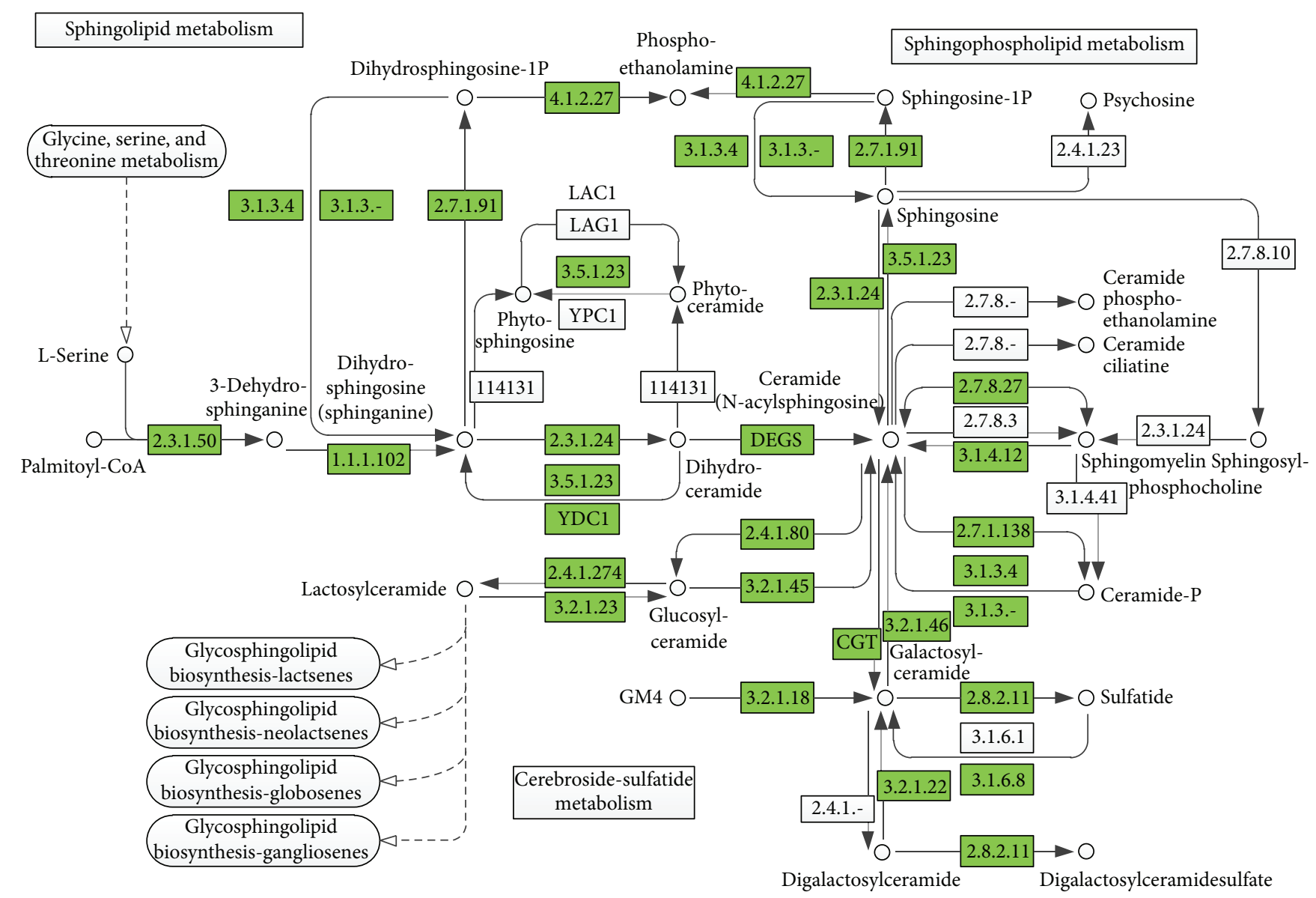

FIGURE 2: System analysis of metabolomic alterations in lung cancer. The KEGG database was searched for each disrupted metabolite detected; each KEGG pathway was scored according to the pathway impact. The map was generated using the KEGG reference map. Green boxes indicate enzymatic activities with putative analogous cases in humans.

and Figure 6 (low levels of biomarkers in the PRLC group). The optimal cutoff points as calculated by Youden's index, sensitivities, specificities, and AUC values are listed in Table 7 (high levels of biomarkers in the PRLC group) and Table 8 (low levels of biomarkers in the PRLC group). The AUC value of GpAEA was 0.916 (95\% CI $=0.847-0.984)$. The optimal cutoff point was 1988.46 , indicating $76.67 \%$ sensitivity and 93.33\% specificity between the POLC and PRLC groups.
The AUC value of sphingosine was 0.966 (95\% CI $=0.911-$ 11.000). The optimal cutoff point was 86.48 , indicating $96.67 \%$ sensitivity and $90.00 \%$ specificity between the PRLC and POLC groups. The AUC values for GpAEA and sphingosine were significantly greater than those of the other eight potential biomarkers, CEA, or CYFRA21-1 and had similar diagnostic value to that of CEA for differentiating PRLC and POLC patients. These data indicate that GpAEA and 
TABLE 2: GC/MS identification of potential serum biomarkers in lung cancer.

\begin{tabular}{|c|c|c|c|c|c|c|}
\hline \multirow[b]{2}{*}{ Number } & \multirow[b]{2}{*}{$\begin{array}{l}\text { Retention } \\
\text { time (min) }\end{array}$} & \multirow[b]{2}{*}{$m / z$} & \multirow[b]{2}{*}{ Metabolites } & \multicolumn{3}{|c|}{ Relative mass intensity } \\
\hline & & & & Control group & $\begin{array}{c}\text { Preoperative lung } \\
\text { cancer group (PRLC) }\end{array}$ & $\begin{array}{c}\text { Postoperative lung } \\
\text { cancer group (POLC) }\end{array}$ \\
\hline 1 & 9.21 & 131.1089 & $\alpha$-Hydroxyisobutyric acid & $306.84 \pm 153.64$ & $556.54 \pm 220.00^{*}$ & $803.58 \pm 329.41^{*}$ \\
\hline 2 & 12.23 & 132.1187 & Serine & $183.48 \pm 96.63$ & $114.92 \pm 89.30^{* \#}$ & $284.16 \pm 184.76$ \\
\hline 3 & 18.99 & 292.2003 & 2,3,4-Trihydroxybutyric acid & $71.89 \pm 30.60$ & $24.63 \pm 24.13^{*}$ & $23.02 \pm 14.47^{*}$ \\
\hline 4 & 30.27 & 122.1582 & 9,12-Octadecadienoic acid & $9.88 \pm 5.79$ & $24.90 \pm 18.09^{* \#}$ & $13.57 \pm 9.30$ \\
\hline 5 & 30.38 & 117.0664 & Oleic acid & $244.99 \pm 131.32$ & $605.66 \pm 361.44^{* \#}$ & $346.58 \pm 164.66$ \\
\hline
\end{tabular}

TABLE 3: Clinical characteristics of subjects at baseline.

\begin{tabular}{lccc}
\hline Samples & Control group & Preoperative lung cancer group (PRLC) & Postoperative lung cancer group (POLC) \\
\hline Sample number & 30 & 30 & 30 \\
Age & $60.35 \pm 12.48$ & $61.58 \pm 10.67$ & $61.58 \pm 10.67$ \\
Sex $(\mathrm{F} / \mathrm{M})$ & $19 / 11$ & $21 / 9$ & $21 / 9$ \\
CEA $(\mathrm{ng} / \mathrm{mL})$ & $1.66 \pm 0.72$ & $3.29 \pm 1.60^{*}$ & $2.24 \pm 1.42$ \\
CYFRA21-1 (ng/mL) & $1.30 \pm 0.46$ & $3.37 \pm 2.66^{*}$ & $1.53 \pm 0.72$ \\
\hline
\end{tabular}

${ }^{*}$ Compared with control group, $p<0.05$; ${ }^{\#}$ compared with postoperative lung cancer group, $p<0.05$.

TABLE 4: Pathway analysis results.

\begin{tabular}{|c|c|c|c|c|c|c|}
\hline & Total & Expected & Hits & Raw $p$ & FDR & Impact \\
\hline Sphingolipid metabolism & 25 & 0.74 & 3 & 0.036 & 0.47 & 0.66 \\
\hline Glycine, serine, and threonine metabolism & 48 & 1.42 & 3 & 0.17 & 0.78 & 0.42 \\
\hline Arginine and proline metabolism & 77 & 2.27 & 5 & 0.074 & 0.66 & 0.27 \\
\hline Galactose metabolism & 41 & 1.21 & 4 & 0.031 & 0.47 & 0.26 \\
\hline Linoleic acid metabolism & 15 & 0.44 & 3 & 0.36 & 1.00 & 0.23 \\
\hline
\end{tabular}

TABLE 5: ROC curves of potential serum biomarker levels for differentiating the control group from the PRLC group.

\begin{tabular}{|c|c|c|c|c|c|c|}
\hline Marker & Cutoff value & Sensitivity (\%) & Specificity (\%) & AUC & $p$ value $^{*}$ & $95 \% \mathrm{CI}^{\mathrm{a}}$ \\
\hline Phosphorylcholine & 78.32 & 90.00 & 80.00 & 0.874 & $<0.001$ & $0.780-0.969$ \\
\hline Glycerophospho-N-arachidonoyl ethanolamine & 1752.60 & 96.67 & 90.00 & 0.983 & $<0.001$ & $0.960-1.007$ \\
\hline$\gamma$-Linolenic acid & 805.17 & 76.67 & 93.33 & 0.889 & $<0.001$ & $0.806-0.972$ \\
\hline$\alpha$-Hydroxyisobutyric acid & 365.23 & 80.00 & 80.00 & 0.860 & $<0.001$ & $0.764-0.956$ \\
\hline 9,12-Octadecadienoic acid & 13.56 & 66.67 & 83.33 & 0.704 & $<0.001$ & $0.562-0.847$ \\
\hline Oleic acid & 402.22 & 70.00 & 86.67 & 0.749 & $<0.001$ & $0.614-0.884$ \\
\hline CEA & 2.54 & 76.67 & 93.33 & 0.867 & $<0.001$ & $0.765-0.969$ \\
\hline CYFRA21-1 & 2.02 & 56.67 & 96.67 & 0.803 & $<0.001$ & $0.690-0.916$ \\
\hline
\end{tabular}

${ }^{*}$ Asymptotic significance, null hypothesis: true area $=0.5$.

${ }^{\mathrm{a}} 95 \%$ confidence interval of the difference.

TABLE 6: ROC curves of potential serum biomarker levels for differentiating the control group from the PRLC group.

\begin{tabular}{|c|c|c|c|c|c|c|}
\hline Marker & Cutoff value & Sensitivity (\%) & Specificity (\%) & AUC & $p$ value ${ }^{*}$ & $95 \% \mathrm{CI}^{\mathrm{a}}$ \\
\hline Prasterone sulfate & 91.07 & 76.67 & 80.00 & 0.787 & $<0.001$ & $0.670-0.905$ \\
\hline Sphingosine & 102.76 & 90.00 & 96.67 & 0.957 & $<0.001$ & $0.894-1.019$ \\
\hline Serine & 113.21 & 83.33 & 73.33 & 0.774 & $<0.001$ & $0.645-0.904$ \\
\hline 2,3,4-Trihydroxybutyric acid & 45.87 & 86.67 & 83.33 & 0.880 & $<0.001$ & $0.794-0.966$ \\
\hline
\end{tabular}

${ }^{*}$ Asymptotic significance, null hypothesis: true area $=0.5$.

${ }^{\mathrm{a}} 95 \%$ confidence interval of the difference. 
TABLE 7: ROC curves of potential serum biomarker levels for differentiating the POLC group from the PRLC group.

\begin{tabular}{lcccccc}
\hline Marker & Cutoff value & Sensitivity (\%) & Specificity (\%) & AUC & $p$ value $^{*}$ & $95 \% \mathrm{CI}^{\mathrm{a}}$ \\
\hline Phosphorylcholine & 84.69 & 76.67 & 70.00 & 0.781 & $<0.001$ & $0.666-0.896$ \\
Glycerophospho-N-arachidonoyl ethanolamine & 1988.46 & 76.67 & 93.33 & 0.916 & $<0.001$ & $0.847-0.984$ \\
$\gamma$-Linolenic acid & 808.24 & 76.67 & 90.00 & 0.847 & $<0.001$ & $0.744-0.949$ \\
9,12-Octadecadienoic acid & 21.29 & 53.33 & 86.67 & 0.645 & $<0.001$ & $0.499-0.791$ \\
Oleic acid & 489.02 & 60.00 & 80.00 & 0.694 & $<0.001$ & $0.550-0.838$ \\
CEA & 2.90 & 70.00 & 83.33 & 0.772 & $<0.001$ & $0.646-0.898$ \\
CYFRA21-1 & 1.64 & 70.00 & 70.00 & 0.737 & $<0.001$ & $0.646-0.863$ \\
\hline
\end{tabular}

*Asymptotic significance, null hypothesis: true area $=0.5$.

${ }^{\mathrm{a}} 95 \%$ confidence interval of the difference.

TABLE 8: ROC curves of potential serum biomarker levels for differentiating the POLC group from the PRLC group.

\begin{tabular}{lcccccc}
\hline Marker & Cutoff value & Sensitivity (\%) & Specificity (\%) & AUC & $p$ value $^{*}$ & $95 \% \mathrm{CI}^{\mathrm{a}}$ \\
\hline Sphingosine & 86.48 & 96.67 & 90.00 & 0.966 & $<0.001$ & 0.911 \\
Serine & 130.97 & 80.00 & 76.67 & 0.825 & $<0.001$ & 0.721 \\
\hline
\end{tabular}

${ }^{*}$ Asymptotic significance, null hypothesis: true area $=0.5$.

${ }^{\mathrm{a}} 95 \%$ confidence interval of the difference.

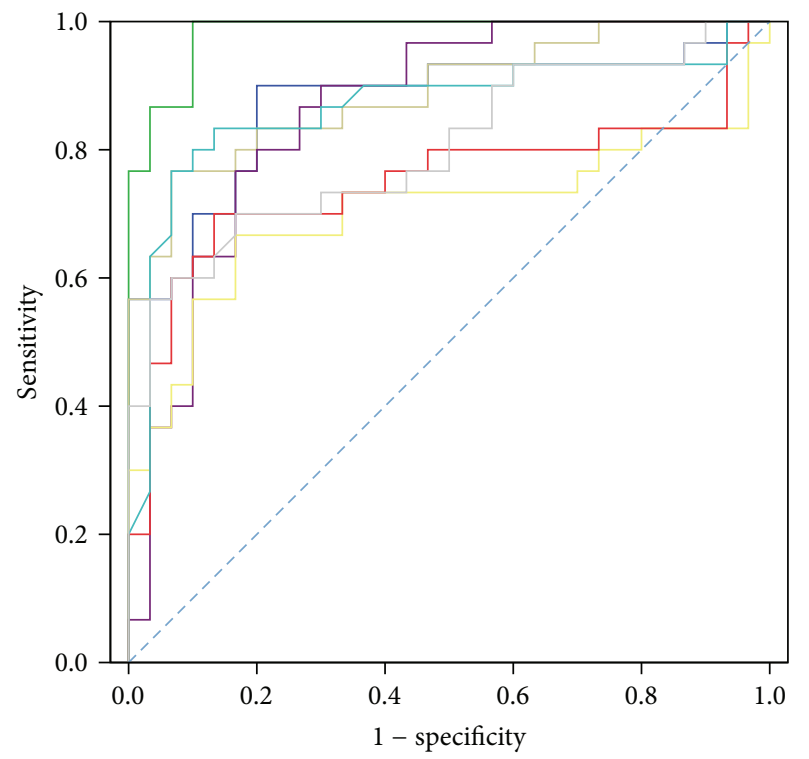

Source of curve

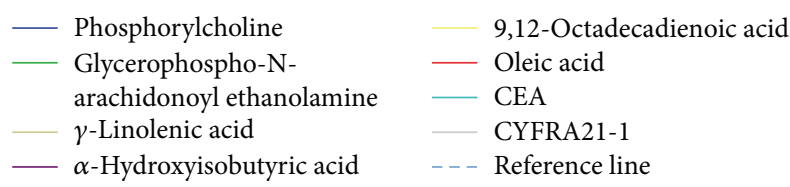

FIGURE 3: ROC curve analysis of potential serum biomarker and other tumor marker (CEA, CYFRA21-1) levels for differentiating the control group from the PRLC group (high levels of biomarkers in PRLC).

sphingosine are high-performance prognostic biomarkers of lung cancer, where high GpAEA levels and low sphingosine levels indicate the risk of lung cancer recurrence.

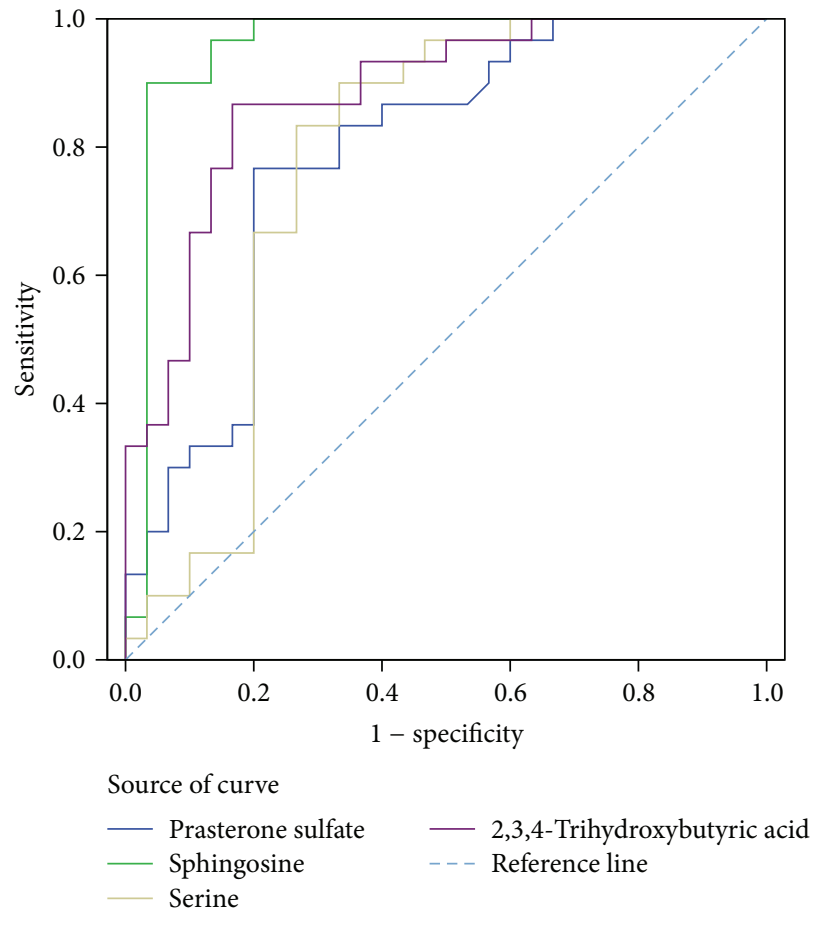

FIGURE 4: ROC curve analysis of potential serum biomarker and other tumor marker (CEA, CYFRA21-1) levels for differentiating the control group from the PRLC group (low levels of biomarkers in PRLC).

\section{Discussion}

With its rates of incidence and death being the highest, lung cancer is the most common malignant tumor worldwide. Despite improvements in lung cancer diagnosis and 


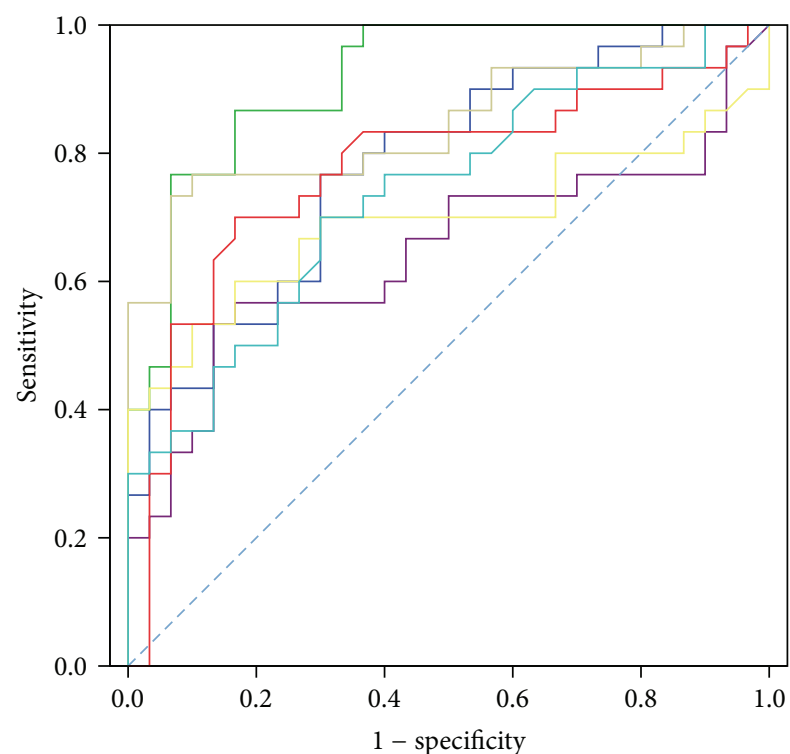

Source of the curve

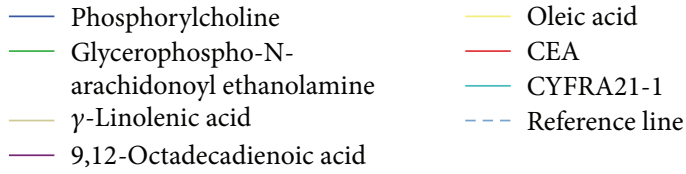

FIgURE 5: ROC curve analysis of potential serum biomarker levels for differentiating the POLC group from the PRLC group (high levels of biomarkers in PRLC).

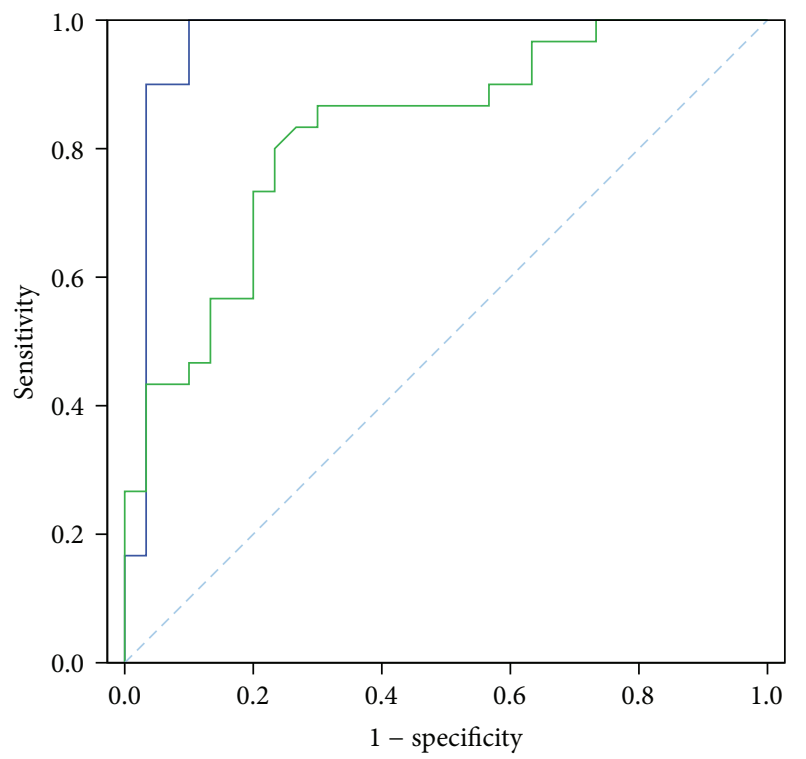

Source of the curve

- Sphingosine

Reference line

FIGURE 6: ROC curve analysis of potential serum biomarker levels for differentiating the POLC group from the PRLC group (low levels of biomarkers in PRLC). treatment in recent years, the rate of 5-year survival rate remains as low as $16 \%$. Identifying tumor markers can potentially improve lung cancer diagnosis, prognostication, and therapy. Biomarkers are conventionally defined as biological molecules that represent health and disease states. They are typically measured in readily available body fluids, lie outside the causal pathway, and can be used to detect and monitor disease burden and response to treatment [15]. Pathway analysis has been used in metabolomics analysis, vastly extending its clinical relevance and effects [16]. However, the metabolic pathways involved in lung cancer have not been well studied. Emerging techniques in metabolomics have provided a powerful platform for the discovery of novel biomarkers and biochemical pathways that can potentially distinguish diseased and healthy subjects.

In this study, we analyzed the construction, interaction, and pathways of potential lung cancer biomarkers using MetPA based on the KEGG database and the Human Metabolome Database and determined that sphingolipid metabolism was the top altered pathway in lung cancer. ROC curve analysis indicated that GpAEA and sphingosine were potential sensitive and specific diagnostic and prognostic biomarkers of lung cancer. Compared with the traditional diagnostic biomarkers of lung cancer, that is, CEA and CYFRA21-1, GpAEA and sphingosine were as good or more appropriate for detecting lung cancer.

Previous studies have demonstrated that sphingolipids such as sphingosine, ceramide, and sphingosine-1-phosphate are important cell membrane components that play an important role in tumorigenesis [17]. Ceramide is a negative regulator of cell proliferation, inhibiting cell proliferation and promoting apoptosis. Conversely, its metabolite sphingosine-1phosphate inhibits apoptosis and promotes cell proliferation. Sphingosine, ceramide, and sphingosine-1-phosphate are mutually transformative and maintain homeostasis through enzymatic reactions. Sphingosine kinase, a major ratelimiting enzyme in the cellular synthesis of sphingosine1-phosphate, regulates both ceramide and sphingosine-1phosphate by reducing ceramide to generate sphingosine1-phosphate. Thus, ceramide and sphingosine-1-phosphate homeostasis determines apoptosis and cell proliferation. Inhibiting sphingosine kinase activity increases ceramide and sphingosine levels and decreases sphingosine-1-phosphate levels, inhibiting cell proliferation and promoting apoptosis [18]. Using HPLC-Q-TOF/MS, Yu et al. [19] found that, compared with healthy volunteers, patients with lung cancer had decreased levels of sphingosine. Consistent with this finding, we previously reported that sphingosine levels were significantly decreased in PRLC patients compared with healthy volunteers and POLC patients [5]. In this study, sphingolipid metabolism was the top altered pathway in lung cancer. Sphingosine, ceramide, and sphingosine-1-phosphate are involved in this metabolic pathway, and the decreased sphingosine levels in the PRLC patients may have led to the decreased ceramide levels and increased sphingosine-1phosphate levels. We believe that the alteration in the levels of these three components in the PRLC patients could have resulted from abnormal activation of the sphingosine kinase pathway, previously implicated in tumor development. 
In our study, GpAEA was the other potential sensitive and specific diagnostic and prognostic biomarker of lung cancer. Previous studies have demonstrated that GpAEA could be hydrolyzed by a metal-dependent phosphodiesterase to produce the archetypal endocannabinoid anandamide (AEA), which belongs to the long-chain lipids [20]. A neurotransmitter, AEA, is rapidly hydrolyzed to arachidonic acid and ethanolamine by fatty acid amide hydrolase, which is present very briefly in the nervous system. AEA has two membrane receptors, brain (CB1-R) and spleen (CB2-R) [21], and mediates cellular signal transduction by activating the receptors. On the other hand, AEA affects the physiological function of cells by disrupting cell membrane lipids. After binding CB1-R, AEA activates sphingomyelinase and hydrolyzes sphingomyelin to generate ceramide [22]. In this study, GpAEA levels were increased in PRLC patients compared with that of the controls and POLC patients. We assume that the increased GpAEA levels lead to decreased AEA production and, subsequently, decreased ceramide production. Due to sphingosine, ceramide, and sphingosine-1-phosphate homeostasis, the decreased ceramide levels could lead to decreased sphingosine levels, which is consistent with our previous results. In our metabolic pathway analysis, sphingolipid metabolism was the top altered pathway in lung cancer. Combined with the above analysis, we believe that GpAEA and sphingosine may both be involved in sphingolipid metabolism and hope that they can be developed as sensitive and specific diagnostic and prognostic biomarkers of lung cancer, which require confirmation in further functional studies and large-sample validation.

In conclusion, we demonstrate that studying metabolomics is a simple and noninvasive approach and may be used for identifying diagnostic and prognostic biomarkers of lung cancer. However, as the sample size in this study was small, further studies involving larger populations of patients with lung cancer should be performed to confirm our findings. These investigations would provide important information on the potential of GpAEA and sphingosine as noninvasive markers of lung cancer.

\section{Conflict of Interests}

The authors declare no conflict of interests regarding the publication of this paper.

\section{Acknowledgments}

Financial support from Zhejiang Province Department of Science \& Technology (2011C23131) is gratefully acknowledged. The authors also thank Shanghai Sensichip Infotech Co., Ltd., for assisting with the bioinformation analysis.

\section{References}

[1] R. Siegel, J. Ma, Z. Zou, and A. Jemal, "Cancer statistics, 2014," CA: A Cancer Journal for Clinicians, vol. 64, no. 1, pp. 9-29, 2014.

[2] R. Molina, J. M. Auge, J. M. Escudero et al., "Mucins CA 125, CA 19.9, CA 15.3 and TAG-72.3 as tumor markers in patients with lung cancer: comparison with CYFRA 21-1, CEA, SCC and NSE," Tumor Biology, vol. 29, no. 6, pp. 371-380, 2008.

[3] R. Xue, Z. Lin, C. Deng et al., "A serum metabolomic investigation on hepatocellular carcinoma patients by chemical derivatization followed by gas chromatography/mass spectrometry," Rapid Communications in Mass Spectrometry, vol. 22, no. 19, pp. 3061-3068, 2008.

[4] M. Bogdanov, W. R. Matson, L. Wang et al., "Metabolomic profiling to develop blood biomarkers for Parkinson's disease," Brain, vol. 131, no. 2, pp. 389-396, 2008.

[5] W. M. Claudino, A. Quattrone, L. Biganzoli, M. Pestrin, I. Bertini, and A. Di Leo, "Metabolomics: available results, current research projects in breast cancer, and future applications," Journal of Clinical Oncology, vol. 25, no. 19, pp. 2840-2846, 2007.

[6] W. R. Wikoff, J. A. Gangoiti, B. A. Barshop, and G. Siuzdak, "Metabolomics identifies perturbations in human disorders of propionate metabolism," Clinical Chemistry, vol. 53, no. 12, pp. 2169-2176, 2007.

[7] J. K. Nicholson, J. Connelly, J. C. Lindon, and E. Holmes, "Metabonomics: a platform for studying drug toxicity and gene function," Nature Reviews Drug Discovery, vol. 1, no. 2, pp. 153$161,2002$.

[8] S. Li, H. Liu, Y. Jin, S. Lin, Z. Cai, and Y. Jiang, "Metabolomics study of alcohol-induced liver injury and hepatocellular carcinoma xenografts in mice," Journal of Chromatography B: Analytical Technologies in the Biomedical and Life Sciences, vol. 879, no. 24, pp. 2369-2375, 2011.

[9] V. W.-S. Wong, G. L.-H. Wong, W. C.-W. Chu et al., "Hepatitis B virus infection and fatty liver in the general population," Journal of Hepatology, vol. 56, no. 3, pp. 533-540, 2012.

[10] A. Zhang, H. Sun, and X. Wang, "Power of metabolomics in biomarker discovery and mining mechanisms of obesity," Obesity Reviews, vol. 14, no. 4, pp. 344-349, 2013.

[11] J.-H. Chen, B. M. Enloe, C. D. Fletcher, D. G. Cory, and S. Singer, "Biochemical analysis using high-resolution magic angle spinning NMR spectroscopy distinguishes lipoma-like well-differentiated liposarcoma from normal fat," Journal of the American Chemical Society, vol. 123, no. 37, pp. 9200-9201, 2001.

[12] J. Chen, W. Wang, S. Lv et al., "Metabonomics study of liver cancer based on ultra performance liquid chromatography coupled to mass spectrometry with HILIC and RPLC separations," Analytica Chimica Acta, vol. 650, no. 1, pp. 3-9, 2009.

[13] K. K. Pasikanti, P. C. Ho, and E. C. Y. Chan, "Gas chromatography/mass spectrometry in metabolic profiling of biological fluids," Journal of Chromatography B: Analytical Technologies in the Biomedical and Life Sciences, vol. 871, no. 2, pp. 202-211, 2008.

[14] Y. Chen, Z. Ma, A. Li et al., "Metabolomic profiling of human serum in lung cancer patients using liquid chromatography/hybrid quadrupole time-of-flight mass spectrometry and gas chromatography/mass spectrometry," Journal of Cancer Research and Clinical Oncology, vol. 141, no. 4, pp. 705-718, 2015.

[15] E. G. Armitage and C. Barbas, "Metabolomics in cancer biomarker discovery: current trends and future perspectives," Journal of Pharmaceutical and Biomedical Analysis, vol. 87, pp. 1-11, 2014.

[16] B. Ganter, N. Zidek, P. R. Hewitt, D. Müller, and A. Vladimirova, "Pathway analysis tools and toxicogenomics reference databases for risk assessment," Pharmacogenomics, vol. 9, no. 1, pp. 35-54, 2008.

[17] S. A. Saddoughi, P. Song, and B. Ogretmen, "Roles of bioactive sphingolipids in cancer biology and therapeutics," in Lipids in 
Health and Disease, vol. 49 of Subcellular Biochemistry, pp. 413440, Springer, Amsterdam, The Netherlands, 2008.

[18] O. Cuvillier, "Downregulating sphingosine kinase-1 for cancer therapy," Expert Opinion on Therapeutic Targets, vol. 12, no. 8, pp. 1009-1020, 2008.

[19] X. Yu, Q. Wu, W. Lu et al., "Metabonomics study of lung cancer cells based on liquid chromatography-mass spectrometry," Se $P u$, vol. 31, no. 7, pp. 691-696, 2013.

[20] D. R. Sagar, A. G. Gaw, B. N. Okine et al., "Dynamic regulation of the endocannabinoid system: implications for analgesia," Molecular Pain, vol. 5, article 59, 2009.

[21] R. Mechoulam, E. Fride, and V. D. Marzo, "Endocannabinoids," European Journal of Pharmacology, vol. 359, no. 1, pp. 1-18, 1998.

[22] M. Guzmán and C. Sánchez, "Effects of cannabinoids on energy metabolism," Life Sciences, vol. 65, no. 6-7, pp. 657-664, 1999. 


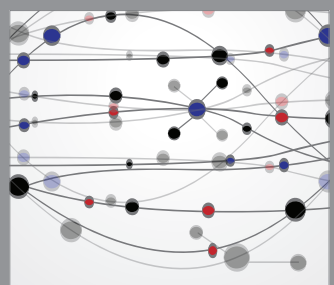

The Scientific World Journal
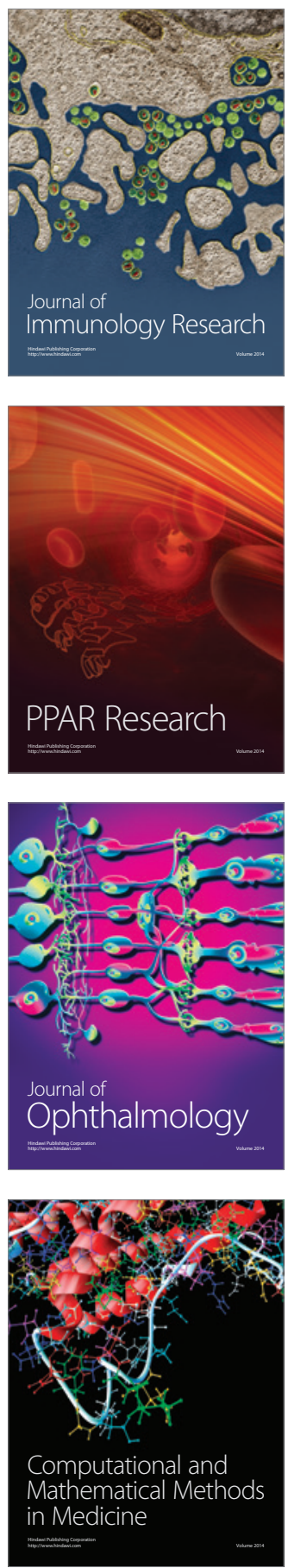

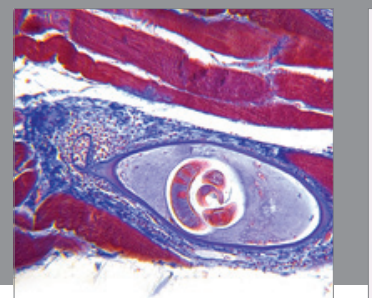

Gastroenterology

Research and Practice
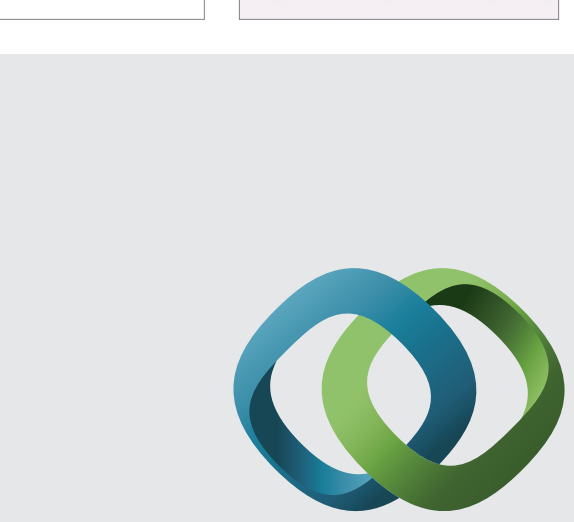

\section{Hindawi}

Submit your manuscripts at

http://www.hindawi.com
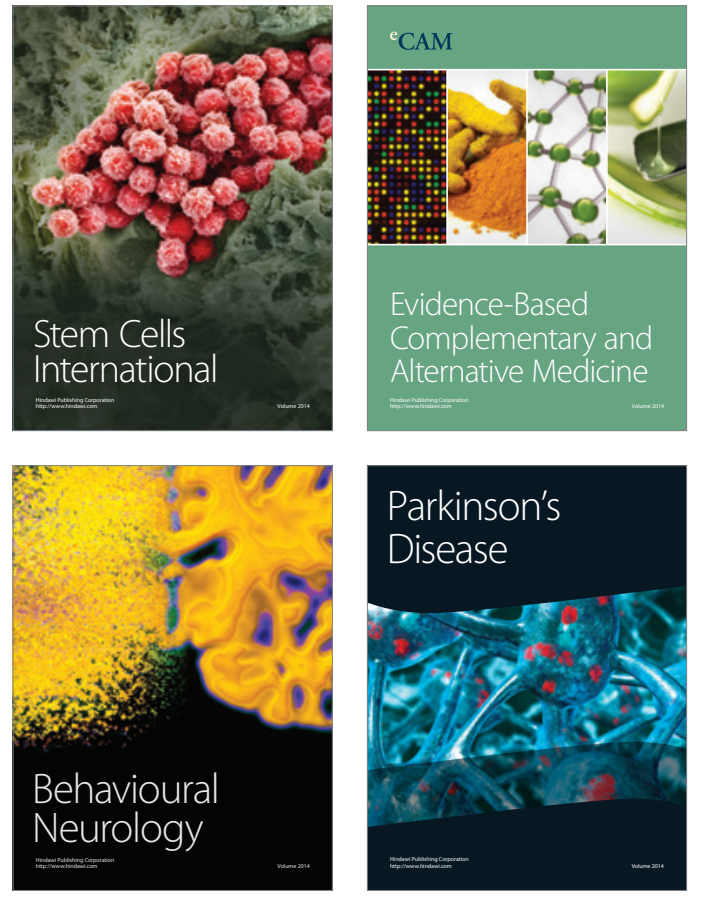
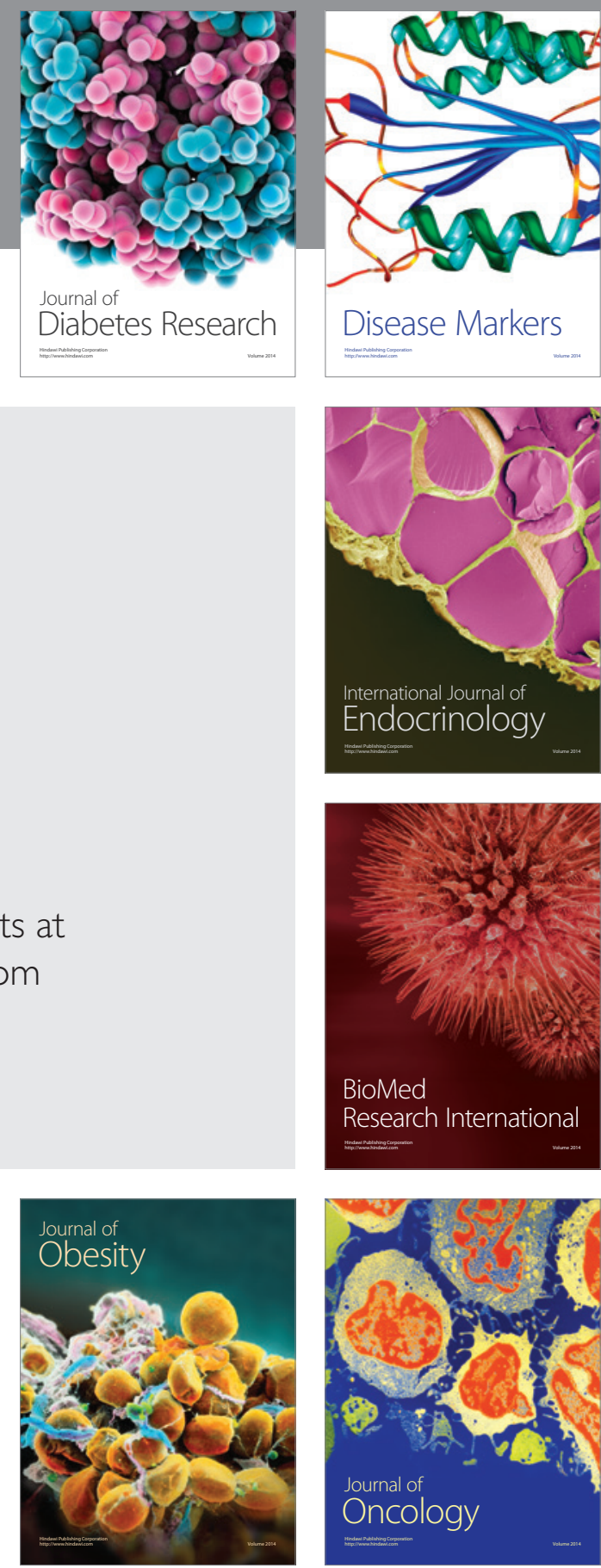

Disease Markers
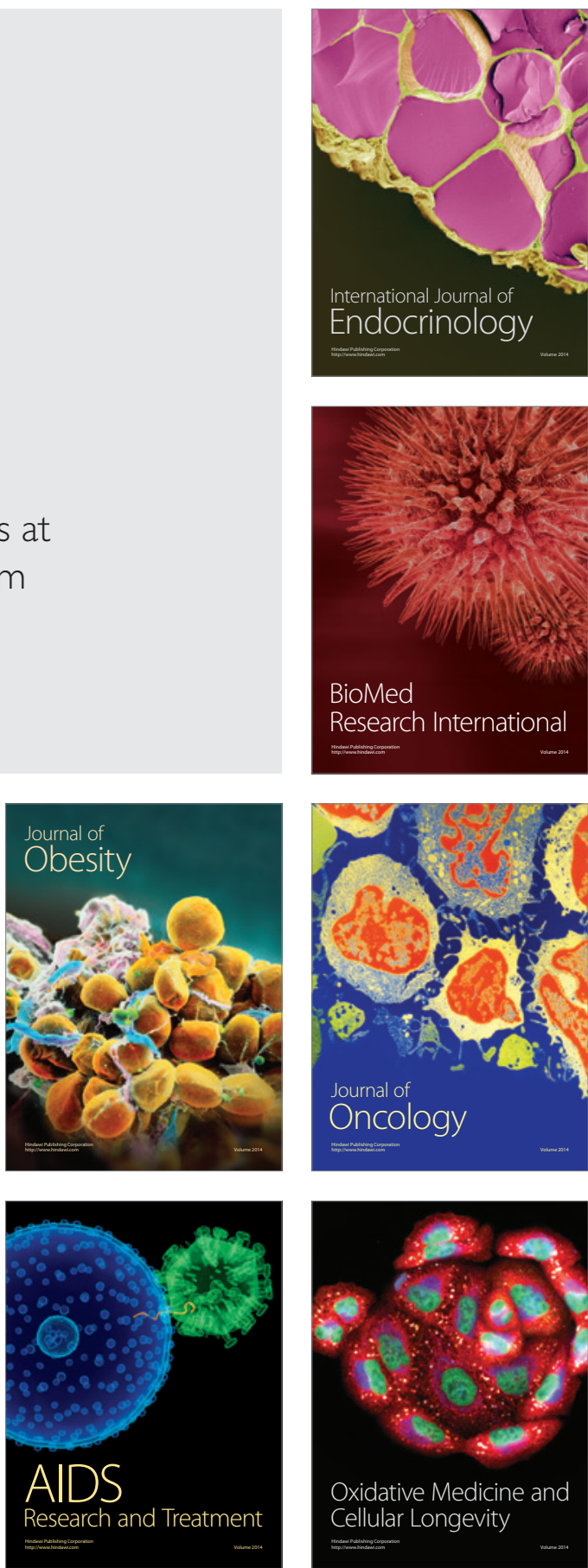